\title{
ANÁLISIS DEL NUEVO TEXTO DEL ARTÍCULO 533 DEL CÓDIGO PROCESAL CIVIL Y LA TERCERÍA DE PROPIEDAD CONTRA HIPOTECAS
}

\author{
Héctor EnRiQue Lama MORE*
}

\begin{abstract}
Resumen
Históricamente, la tercería excluyente de propiedad ha sido un medio de defensa de un tercero cuyos bienes han sido objeto de medidas de embargo o de secuestro dictadas por un juez en un proceso en el que dicha persona no es parte. Ello se encuentra en estricta coincidencia con una correcta interpretación del texto anterior del artículo $533^{\circ}$ del C.P.C, sin embargo, la Sala Civil Permanente de la Corte Suprema de Justicia durante los años que van del 2006 a 2007 y parte del 2008, sostuvo que cuando la referida norma señalaba que la tercería puede fundarse en la propiedad de bienes afectados con medida cautelar o para su ejecución, se debía incluir también en dicho supuesto "la ejecución de garantías reales". Esto motivó la modificación -introducida por Decreto Legislativo $\mathrm{N}^{\circ} 1069$ - del texto del primer párrafo del artículo $533^{\circ}$ del C.P.C., y se introdujo en dicha norma procesal la palabra "judicialmente" a efecto de quitar cualquier duda sobre la naturaleza de la afectación jurídica que recae sobre los bienes que según el tercerista serían de su propiedad.
\end{abstract}

Palabras clave: Tercería de propiedad - Garantías reales - Gravamen hipotecario.

\begin{abstract}
Historically, the intervention of a third party who takes a position contrary to the position of the litigants has constituted a means of defense of a third party, whose properties have been subject to attachment or seizure measures determined by a judge in a proceeding in which said person is not a party. The foregoing strictly coincides with a correct interpretation of the previous text of Article 533 of the Code of Civil Procedures, however, during the years 2006 to 2007 and part of 2008, the Standing Civil Division of the Supreme Court of Justice sustained that when the referred law stipulated that the third party claim could be grounded on the ownership of properties subject to injunction measures or to foreclosure thereof, "the foreclosure of collaterals" should also be included in the referred assumption. This motivated the amendment - introduced by Legislative Decree $\mathrm{N}^{\circ} 1069$ - of the text of the first paragraph of Article $533^{\circ}$ of the Code of Civil Procedures, whereby the word "judicially" was included with the aim of leaving no doubt about the nature of the legal encumbrance of the properties which, according to the intervener, would be owned by him.
\end{abstract}

Key words: Third party or intervener - Collaterals - Mortgage lien.

\footnotetext{
* Juez Superior Titular de la Corte Superior de Justicia de Lima - Poder Judicial de Perú. Ha sido miembro del Consejo Consultivo del Centro de Investigaciones Judiciales.
} 
Héctor Enrique Lama More - Tercería de propiedad contra hipotecas en el Código Procesal Civil

\begin{abstract}
Sumario
1. Antecedentes. 2. Nuevo texto normativo del artículo $533^{\circ}$ del C.P.C. 3. Segundo párrafo del artículo $533^{\circ}$ del C.P.C. 3.1. Duplicidad de partida registral. 3.2. Nulidad o ineficacia del acto jurídico de transferencia de propiedad a favor del que constituyó la hipoteca, y cancelación del asiento de propiedad de este. 4. Finalidad de la tercería de propiedad contra hipoteca. 5. Necesidad de cubrir el vacío normativo.
\end{abstract}

\title{
1. ANTECEDENTES
}

Los pronunciamientos expedidos en los últimos años por las salas civiles de la Corte Suprema de Justicia, respecto de la admisión a tramite o del rechazo liminar de las demandas de tercerías de propiedad contra hipotecas fueron realmente contradictorios.

Ello se pudo apreciar en diversas resoluciones expedidas en Casación; solo a modo de ejemplo se pueden señalar dos de ellas. Es el caso de los pronunciamientos contradictorios que se presentaron entre la Casación No 5329-2006-LIMA, de fecha 27 de marzo del 2007, expedida por la Sala Civil Permanente de la Corte Suprema de la República, y la Casación $\mathrm{N}^{\mathrm{O}}$ 2360-2006-LIMA, de fecha 21 de Marzo del 2007, expedida por la Sala Civil Transitoria de la Corte Suprema de Justicia. En la primera de ellas se estableció -apartándose de criterios jurisprudenciales expedidos anteriormente- que cuando el artículo $533^{\circ}$ del C.P.C. ${ }^{1}$, en su texto vigente en ese momento, señalaba que la tercería puede fundarse en la propiedad de bienes afectados con medida cautelar o para su ejecución, se incluye en dicho supuesto "la ejecución de garantías reales"; ordenando en tal caso la admisión a trámite de la demanda. Por su parte, la otra Ejecutoria señaló en su oportunidad que la pretensión contenida en una demanda de tercería excluyente de propiedad contra una hipoteca contenía un petitorio que resulta jurídicamente imposible, y que, como tal, debía ser rechazada liminarmente ${ }^{2}$.

Tales pronunciamientos causaron gran preocupación entre jueces especializados y jueces superiores, dada la trascendencia del tema y el impacto de tales decisiones en nuestro medio. Ello originó que el tema sea llevado a diversos eventos organizados al interior del Poder Judicial con el objeto de unificar criterios jurisprudenciales. Tres eventos académicos y jurisprudenciales realizados entre jueces superiores durante el año 2008, incluyeron el tema en sus respectivas agendas. 
Héctor Enrique Lama More - Tercería de propiedad contra hipotecas en el Código Procesal Civil

Estos eventos fueron ${ }^{3}$ :

a.- El Pleno Jurisdiccional Regional Civil realizado en la ciudad de Arequipa, los días 28 y 29 de marzo del 2008, al que concurrieron jueces superiores de los distritos judiciales ubicados en el Sur del país, como son los de Arequipa, Cuzco, Puno, Madre de Dios, Apurimac, Moquegua y Tacna.

En dicho Pleno se acordó por mayoría, es decir, por 29 contra 11 votos, que se debe rechazar liminarmente la demanda de tercería de propiedad contra derechos reales de garantía, en razón de que el petitorio constituye un imposible jurídico.

b.- El Pleno Jurisdiccional Regional Civil y Contencioso Administrativo, realizado en la ciudad de Trujillo, los días 18 y 19 de abril del 2008, al que concurrieron jueces superiores de los distritos judiciales del norte del país, como son los de La Libertad, Ancash, del Santa, Cajamarca, Lambayeque, Piura y Tumbes.

En el mencionado pleno jurisdiccional, el total de asistentes adoptaron una sola posición, es decir, por unanimidad se acordó que "Es procedente el rechazo liminar de la demanda de tercería de propiedad interpuesta contra procesos de ejecución de garantías, por causal de imposibilidad jurídica del petitorio".

c.- El Pleno Jurisdiccional Nacional Civil, realizado en la ciudad de Lima los días 06 y 07 de junio del 2008, al que concurrieron jueces superiores de los distritos judiciales de todo el país.

En dicho evento, por 71 a 12 votos - con 03 abstenciones - , el Pleno Jurisdiccional Nacional Civil adoptó, por mayoría, el siguiente acuerdo: "se debe rechazar liminarmente la demanda de tercería de propiedad interpuesta contra la ejecución de garantías reales, porque el petitorio constituye un imposible jurídico"

La controversia no era de reciente data, pues como se sabe el tema fue incluido como punto de agenda al IV Pleno Jurisdiccional Civil realizado en la ciudad de Tacna entre el 23 y el 26 de agosto del año 2000 a la que concurrieron más de medio centenar de jueces superiores de los 25 distritos judiciales de la República ${ }^{4}$. En dicho pleno jurisdiccional se acordó por mayoría (36 votos) que: no procede la tercería de propiedad contra gravamen que proviene de garantía real. Una minoría (14 votos) sostuvo que si resultaba procedente la tercería contra gravamen proveniente de garantía real, independientemente de que se declare fundada o infundada la pretensión contenida en la demanda ${ }^{5}$. 
Héctor Enrique Lama More - Tercería de propiedad contra hipotecas en el Código Procesal Civil

Como se puede apreciar, la jurisprudencia de los órganos jurisdiccionales especializados y superiores en lo Civil -incluida la sub-especialidad Comercial en Lima - adoptó mayoritariamente una posición distinta - y discrepante - con la línea jurisprudencial adoptada por la Sala Civil Permanente de la Corte Suprema de Justicia durante los años que van del 2006 a 2007 y parte del 2008.

\section{NUEVO TEXTO NORMATIVO DEL ARTÍCULO $533^{\circ}$ DEL CÓDIGO PROCESAL CIVIL}

El 28 de junio del 2008, se publicó en el diario oficial "El Peruano" el Decreto Legislativo $\mathrm{N}^{\circ}$ 1069. Dicha norma, recogiendo la inquietud mayoritaria de los magistrados reflejada en los plenos jurisdiccionales citados, incluye la modificación del artículo $533^{\circ}$ del C.P.C. ${ }^{6}$, a efecto de dejar establecido, sin lugar a dudas, que la demanda de tercería de propiedad solo resultaba procedente cuando esta se sustenta en la propiedad de bienes afectados por mandato judicial y no como consecuencia de un acto jurídico originado o realizado por particulares, o por acuerdo privado, como es el caso de las hipotecas - unilaterales, voluntarias o legales.

En realidad, históricamente la tercería excluyente de propiedad ha sido un medio de defensa del tercero cuyos bienes han sido objeto de medidas de embargo o de secuestro dictadas por un juez en un proceso donde dicha persona no es parte. Si el juez advierte que el bien afectado con medida cautelar no pertenece al deudor demandado corresponde se disponga la inmediata desafectación.

Una correcta interpretación del texto anterior del artículo $533^{\circ}$ del C.P.C., permitía apreciar que cuando en él se señalaba que la tercería puede sustentarse en la propiedad de bienes afectados con medida cautelar o para la ejecución, en este último supuesto debía entenderse, como así lo entendió la mayoría de los órganos jurisdiccionales del país, con excepción de la Sala Civil Permanente de la Corte Suprema como se ha indicado líneas arriba, que se trataba de la propia medida cautelar dictada por el juez, pero que se encontraba en la fase de ejecución forzada y no de una afectación jurídica de carácter extrajudicial, originada en un acto jurídico unilateral o bilateral, como son los gravámenes constituidos por garantías reales.

Pese a ello el nuevo texto - introducido por el Decreto Legislativo No 1069 del primer párrafo del artículo $533^{\circ}$ del C.P.C., ha permitido superar la confusión originada en pronunciamientos de una de las salas civiles del máximo 
Héctor Enrique Lama More - Tercería de propiedad contra hipotecas en el Código Procesal Civil

tribunal de la República, introduciendo en dicha norma procesal la palabra "judicialmente" a efecto de quitar cualquier duda sobre la naturaleza de la afectación jurídica que recae sobre los bienes que según el tercerista serían de su propiedad.

La precisión era urgente, dada la trascendencia de la decisión final que debía ser adoptada.

Así, por ejemplo, si en un proceso de ejecución de garantía hipotecaria, un tercero, invocando haber adquirido la propiedad del bien materia de ejecución, pero que no inscribió de modo oportuno su derecho de propiedad, permitiendo que el anterior propietario hipoteque el bien cuando ya no era de su propiedad, interponía tercería de propiedad con el objeto de que se deje sin efecto el gravamen hipotecario o que se priven los efectos del citado gravamen registral; el juez, al calificar la demanda podía optar entre admitir la demanda o rechazarla liminarmente, dependiendo ello de la interpretación que le daba al anterior texto del artículo $533^{\circ}$ del C.P.C.; si el juez consideraba que en tal caso se encontraba frente a un bien afectado con una "medida" para la ejecución, y entendía que en ella se incluía la ejecución de garantías reales, admitía a trámite la demanda, suspendiendo con ello la ejecución hipotecaria hasta la conclusión de dicho proceso, lo que podía ocurrir transcurrido dos o tres años.

En tal caso, el resultado de dicho proceso no podía ser exitoso para el tercerista, pues el derecho real del acreedor hipotecario siempre prevalecerá frente al derecho real de propiedad del tercerista, teniendo en cuenta que el derecho del acreedor hipotecario se inscribió con anterioridad al derecho del tercerista - primer párrafo del artículo $2022^{\circ}$ del C.C. En todo caso, la existencia de buena o mala fe del que tiene el derecho real primeramente inscrito no corresponde ventilarse en un proceso de tercería de propiedad. Por lo demás, solo podía jurídicamente privarse los efectos al gravamen hipotecario, dejando sin efecto el acto jurídico que le dio origen, en un proceso distinto al de tercería.

La otra opción que tenía el juez era la de rechazar liminarmente la demanda con el argumento de que la norma procesal - citada en el párrafo precedente - dispone que la tercería de propiedad solo procede cuando los bienes del tercerista han sido afectados por medida judicial, no resultando posible jurídicamente cuando la afectación se ha originado en gravamen extrajudicial, como la hipoteca -inciso $6^{\circ}$ del artículo $427^{\circ}$ del C.P.C. Esta fue la postura de la mayoría de los órganos jurisdiccionales, como se ha indicado líneas arriba. 
Héctor Enrique Lama More - Tercería de propiedad contra hipotecas en el Código Procesal Civil

En este último caso, no habiéndose admitido a trámite la demanda, el proceso de ejecución continuaba su trámite, ejecutándose la subasta, adjudicando el bien al mejor postor, disponiendo la transferencia de propiedad al adjudicatario e inscribiendo su derecho en el Registro de Propiedad Inmueble respectivo, cancelando todos los gravámenes y ordenando el lanzamiento del bien subastado. Sin embargo, al cabo de dos o tres años, cuando el proceso principal se encontraba concluido, una resolución de una de las salas civiles de la Corte Suprema de Justicia disponía que la demanda de tercería de propiedad debía ser admitida a trámite; tal decisión llegaba muchas veces cuando el bien se encontraba enajenado a terceros y la suma recaudada se había entregado al acreedor demandante. Tremendo problema para el juez de la causa, pues, ni el acreedor estaba dispuesto a devolver lo ya cobrado, ni el nuevo dueño estaba dispuesto a "devolver " el bien que adquirió del adjudicatario en virtud de la fe registral.

Con el nuevo texto del artículo $533^{\circ}$ del C.P.C. - primer párrafo - se puso fin a dicha incertidumbre.

En todo caso, el derecho del tercero afectado con un ilegal gravamen hipotecario corresponde hacerse valer en un proceso especialmente dirigido a lograr la privación total de los efectos del acto jurídico que dio origen al citado gravamen, sea por nulidad, anulabilidad o ineficacia, pudiendo en dicho proceso asegurar su pretensión con una medida cautelar de anotación de demanda, medida cautelar, dígase de paso, que no se cancela por efecto de la adjudicación en subasta pública.

\section{SEGUNDO PÁRRAFO DEL ARTÍCULO 533 DEL CÓDIGO PROCESAL CIVIL}

El Decreto Legislativo $N^{\circ} 1069$ introdujo una figura nueva - en nuestro medio - en el ámbito de las tercerías de propiedad, agregando un segundo párrafo a la citada norma procesal, cuyo texto es el siguiente:

"Sin perjuicio de lo señalado, puede fundarse en la propiedad de bienes afectados con garantías reales, cuando el derecho del tercerista se encuentra inscrito con anterioridad a dicha afectación."

La citada norma procesal no es una "creación" o "invento" del legislador peruano, pues un texto parecido aparece en la legislación española ${ }^{7}$. 
Héctor Enrique Lama More - Tercería de propiedad contra hipotecas en el Código Procesal Civil

En materia de tercería de propiedad, la citada norma española establece, de modo excepcional, la procedencia de la tercería de propiedad contra hipotecas, imponiendo como requisito que el derecho de propiedad del tercerista se encuentre inscrito con anterioridad al gravamen hipotecario.

Es de suponer que el derecho del tercerista quedará a buen recaudo si su derecho de propiedad es anterior al del gravamen hipotecario; es aplicable en este caso el aforismo latino muy antiguo, de relevante vigencia en la actualidad -en especial en materia registral - e incorporado en las normas sustantivas civiles: "prior tempore potior iure", es decir, primero en el tiempo mejor en el derecho.

La introducción de tal figura en nuestra norma procesal no constituye, en modo alguno, una regulación "inútil" y "carente de sentido", y que podría quedar abrogado por el desuso; pues podría pensarse que resulta bastante difícil - por no decir imposible - que el derecho del tercerista se encuentre inscrito con anterioridad al del acreedor hipotecario, pues el registrador no podría haber inscrito la hipoteca si no se verifica que el constituyente aparezca como propietario registral.

Sin embargo, la experiencia judicial ha puesto sobre la mesa algunos casos que han tenido que ser enfrentados jurisdiccionalmente con bastante dificultad.

Expondremos a modo de ejemplo solo dos supuestos a los que podría aplicarse, de modo excepcional el actual texto contenido en el segundo párrafo del artículo $533^{\circ}$ del C.P.C.

\subsection{Duplicidad de partida registral}

Es el caso de aquella persona propietaria registral de un inmueble, que toma conocimiento que dicho bien de su propiedad será sometido a subasta pública en ejecución de una hipoteca que él no ha constituido y que se encuentra inscrita con posterioridad a su derecho de propiedad, pero en una partida registral distinta a la suya, es decir, paralela, abierta en virtud a un titulo supletorio. En dicha partida registral paralela aparece como propietario el ejecutado en el proceso de ejecución de garantías, quien constituyó la hipoteca materia de ejecución.

El propietario registral en cuya partida, no aparece gravamen alguno, inscribió su derecho con anterioridad al derecho del constituyente de la hipoteca; el gravamen aparece en partida registral distinta. Dos partidas registrales abiertas y vigentes respecto de un mismo inmueble. 
Por supuesto, estamos frente a un caso de excepción que merece tutela urgente, máxime cuando tal irregularidad registral no es poco común en nuestro país, pues se conoce que en muchos lugares del país, incluido Lima, se han llegado a presentar hasta tres o cuatro partidas registrales paralelas respecto de un mismo bien. Es evidente que en este caso el derecho del acreedor hipotecario - con derecho real inscrito - prevalecerá frente al derecho de propiedad no inscrito del tercero, en virtud de lo dispuesto en el primer párrafo del artículo $2022^{\circ}$ del C.C., que se sustenta en el aforismo latino citado anteriormente. En este caso la excepción confirma la regla. En tal situación excepcional creo que corresponde admitir a trámite la demanda de tercería de propiedad en virtud a la norma procesal citada.

\subsection{Nulidad o ineficacia del acto jurídico de transferencia de propiedad a favor del que constituyó la hipoteca, y cancelación del asiento de propiedad de este.}

Otro supuesto es el que se presenta cuando, en una misma partida registral, una persona de nombre A logra, mediante sentencia firme, privar por completo la eficacia del acto jurídico de transferencia de propiedad a favor de otra persona de nombre B. En dicha transferencia A aparece como transferente. Como consecuencia se cancela el asiento registral que consignaba dicha transferencia.

Sin embargo, en el rubro gravámenes de la partida registral del mismo inmueble aparece un asiento en la que B ha constituido hipoteca a favor de C cuando aún tenía inscrito el bien a su nombre. Dicho gravamen mantiene su eficacia.

En este caso, A, quien es propietario registral con derecho vigente, no ha constituido la hipoteca inscrita y no es parte en el proceso de ejecución de citada garantía real que ha iniciado $C$ frente al incumplimiento de la obligación garantizada con la hipoteca constituida por B.

Si A presenta demanda de tercería de propiedad a efecto de que se suspenda la subasta del bien de su propiedad, convocada en el proceso de ejecución iniciado por $C$, cumpliría los requisitos de admisibilidad previstos en el segundo párrafo del artículo $533^{\circ}$ del C.P.C. Estaríamos frente a un tercerista cuyo derecho de propiedad se ha inscrito con anterioridad a la inscripción del gravamen hipotecario. 
Héctor Enrique Lama More - Tercería de propiedad contra hipotecas

en el Código Procesal Civil

\section{FINALIDAD DE LA TERCERÍA DE PROPIEDAD CONTRA HIPOTECA}

Según se aprecia, la finalidad del segundo párrafo del artículo $533^{\circ}$ del C.P.C., es proporcionar de modo urgente, una medida inmediata y eficaz al propietario registral que no constituyó la hipoteca.

Efectivamente, como se ha indicado, el único supuesto que nuestra norma procesal prevé para admitir a trámite una demanda de tercería de propiedad contra gravamen hipotecario es cuando el derecho de propiedad del tercerista se encuentra inscrito con anterioridad a la afectación. El efecto inmediato es la suspensión de la subasta.

Pues bien, en tal caso surgen de inmediato diversas interrogantes, como por ejemplo: ¿qué se busca con la referida tercería de propiedad?, ¿el objeto de esta demanda es levantar el gravamen o solo suspender la ejecución?

La norma procesal no establece de modo expreso alguna respuesta, por lo que frente al vacío normativo corresponde esbozar algunas ideas en relación a este asunto, por tratarse de un tema de relevante importancia.

Reflexionando sobre este tema con algunos colegas, conveníamos en que la tercería de propiedad prevista, en general, en las normas procesales, no está pensada para ser utilizada en los casos de afectaciones originadas en cautelas extrajudiciales, como es el caso de las hipotecas, sino para aquellas afectaciones originadas en un mandato judicial, pues siendo el juez de la medida el competente para conocer este proceso, solo él puede dejar sin efecto una afectación ordenada por su propio despacho, al verificar que el bien no pertenece al deudor sino a un tercero.

Sin embargo, habiendo el legislador incluido en la norma procesal la posibilidad jurídica de utilizar el proceso de tercería para detener la ejecución de un gravamen hipotecario - o de garantía real en general - , resulta necesario comentar sobre los alcances y efectos de este proceso, en caso la pretensión del tercerista sea estimada.

En principio creo que el proceso de tercería de propiedad no resultaría ser la vía idónea para disponer el levantamiento del gravamen hipotecario, pues este se ha originado en un acto jurídico - constitución de garantías reales - cuya validez no ha sido objeto de debate en el proceso de tercería, y no resultaría válido jurídicamente privar los efectos de un asiento registral, manteniendo 
Héctor Enrique Lama More - Tercería de propiedad contra hipotecas en el Código Procesal Civil

vigente el acto jurídico contenido en el título registral que le dio origen; el asiendo registral es el efecto y el acto jurídico es la causa.

La suspensión de la ejecución hipotecaria dispuesta en el admisorio de la demanda, constituye, en mi opinión, en estricto una medida provisoria, similar a una medida cautelar de no innovar, con la característica especial de que su dictado es efecto inmediato y obligatorio de la admisión de la demanda; es decir, se trata de un mandato judicial mediante el cual se dispone, de modo temporal, la no ejecución de la subasta o remate público hasta que se defina, en sentencia firme, si la pretensión del tercerista corresponde ser o no atendida. Si se ampara la demanda del tercerista mediante sentencia firme, esta deberá - en mi opinión - disponer se mantenga en suspensión, hasta que en un proceso en forma - ordinario o de amplia cognición - , se dilucide y defina en sentencia la validez - o invalidez - del acto jurídico de constitución de la garantía real.

¿Quién deberá iniciar ese otro proceso - ordinario o de amplia cognición -? ¿El tercerista o el acreedor hipotecario? Creo que cualquiera de ellos. Si la inicia el tercerista, resulta obvio que su pretensión será la de invalidez del acto jurídico de constitución de la garantía real; y si la inicia el acreedor hipotecario, su pretensión podría ser la de confirmar la validez del citado acto jurídico; en este último caso, en razón de que los efectos de dicho acto jurídico quedaron cuestionados al tener el tercerista su derecho inscrito con anterioridad al gravamen hipotecario.

\section{NECESIDAD DE CUBRIR EL VACÍO NORMATIVO}

Lo expuesto líneas arriba - la necesidad de recurrir a otro proceso - , podría evitarse, si se establece normativamente que en el caso previsto en el segundo párrafo del artículo $533^{\circ}$ del C.P.C., por excepción, la tercería de propiedad puede acumularse con la pretensión de invalidez o ineficacia del acto jurídico de constitución de la garantía real, de tal manera que en un solo proceso se pueda definir no solo la eficacia del asiendo registral que contiene el gravamen hipotecario, sino además la validez - o invalidez - del acto jurídico contenido en título registral que dio origen a la afectación del bien.

Con ello, de ampararse la pretensión del tercerista en todos sus extremos, se declararía la invalidez de la hipoteca o garantía real, y además, la cancelación del gravamen. Por el contrario, si se desestima la demanda del tercerista, se continuará con la ejecución de la garantía real que había quedado en suspenso con la admisión a trámite de la demanda. 
Héctor Enrique Lama More - Tercería de propiedad contra hipotecas en el Código Procesal Civil

1 Artículo $533^{\circ}$ del C.P.C. (texto vigente hasta el 28 de junio del 2008): “La tercería se entiende con el demandante y el demandado, y sólo puede fundarse en la propiedad de los bienes afectados por medida cautelar o para la ejecución; o en el derecho preferente a ser pagado con el precio de tales bienes".

2 Lama More, Héctor Enrique. "Comentarios y reflexiones acerca de las tercerías de propiedad contra hipotecas y garantías reales". Revista Oficial del Poder Judicial, Corte Suprema de Justicia de la República. 2007, № 2, Lima: Centro de Investigaciones Judiciales del Poder Judicial y Proyecto de apoyo a la Reforma del Sistema de Justicia del Perú - JUSPER, p. 118.

3 Disponible en internet: http://www.pj.gob.pe

4 Durante la segunda mitad de la década de los noventa se realizaron en el Poder Judicial plenos jurisdiccionales en todas las especialidades - civil, penal, laboral, familia. Eran eventos de discusión y debate, y fueron convocados por la entonces Comisión Ejecutiva del Poder Judicial. A ellos concurrieron jueces superiores de todos los distritos judiciales de la República, con el objeto de uniformizar criterios, respecto de aquellos casos que conocían los órganos jurisdiccionales, en donde se hayan dado respuestas diversas o contradictorias. El Primer Pleno Jurisdiccional Civil se realizó en la ciudad de Trujillo en 1997, el Segundo se llevó adelante en la ciudad Piura, en agosto de 1998, el Tercero se realizó en la ciudad de Cusco, en septiembre de 1999 y el último se llevó adelante en la ciudad de Tacna, en agosto del 2000.

5 En el citado pleno jurisdiccional, los magistrados esbozaron diversos argumentos, cada uno defendiendo sus respectivos puntos de vista. El autor de estas líneas participó en el citado evento adhiriéndose a la posición mayoritaria. No existe publicación oficial del Poder Judicial sobre los acuerdos adoptados en dicha reunión, sin embargo, se conocen sus resultados y han sido objeto de diversas publicaciones, así por ejemplo, la "Guía Rápida de Jurisprudencia Vinculante y Acuerdos de Plenos Jurisdiccionales", publicada por Gaceta Jurídica Sociedad Anónima, en Octubre del año 2002, incluye información sobre dichos acuerdos.

6 Artículo $533^{\circ}$ del C.P.C. (modificado por el D. Leg. 1069, publicado el 28 de Junio del 2008).- “La tercería se entiende con el demandante y el demandado, y sólo puede fundarse en la propiedad de los bienes afectados judicialmente por medida cautelar o para la ejecución; o en el derecho preferente a ser pagado con el precio de tales bienes.

Sin perjuicio de lo señalado, puede fundarse en la propiedad de bienes afectados con garantías reales, cuando el derecho del tercerista se encuentra inscrito con anterioridad a dicha afectación." (resaltado nuestro)

7 Ley de Enjuiciamiento Civil (LEC) 1/2000 - España - Artículo $696^{\circ}$ (tercerías de dominio).

1. Para que pueda admitirse la tercería de dominio en los procedimientos a que se refiere este capítulo, deberá acompañarse a la demanda título de propiedad de fecha fehaciente anterior a la de constitución de la garantía. Si se tratare de bienes cuyo dominio fuere susceptible de inscripción en algún registro, dicho título habrá de estar inscrito a favor del tercerista o de su causante con fecha anterior a la de inscripción de la garantía, lo que se acreditará mediante certificación registral expresiva de la inscripción del título del tercerista o de su causante y certificación de no aparecer extinguido ni cancelado en el registro el asiento de dominio correspondiente.

2. La admisión de la demanda de tercería suspenderá la ejecución respecto de los bienes a los que se refiera y, si estos fueren solo parte de los comprendidos en la garantía, podrá seguir el procedimiento respecto de los demás, si así lo solicitare el acreedor. 